\title{
Comparison of beam combiners for a synthetic aperture imaging telescope
}

\section{Casper van der Avoort}

\section{Silvania F. Pereira}

Joseph J.M. Braat

Jan-Willem den Herder

\author{
Delft University of Technology, Optics Research Group, Lorentzweg 1, NL-2628 CJ, Delft, The Nether- \\ lands \\ Delft University of Technology, Optics Research Group, Lorentzweg 1, NL-2628 CJ, Delft, The Nether- \\ lands \\ Delft University of Technology, Optics Research Group, Lorentzweg 1, NL-2628 CJ, Delft, The Nether- \\ lands \\ SRON, Netherlands Institute for Space Research, Sorbonnelaan 2, NL-3584 CA, Utrecht, The Nether- \\ lands
}

Future astronomical space missions will comprise a constellation of several optical telescopes to detect exo-planets by interferometric nulling of starlight. The Darwin mission of the European Space Agency (ESA) and NASAs Terrestrial Planet Finder Interferometer both consist of a free-flying collection of telescopes and a beam combiner. As such, the constellation provides a co-phased array of telescopes that can also be used for aperture synthesis imaging. This imaging technique relies on recording intensity interference patterns, in which the layout of the beam combination optics and the detector play a key role. Several designs for beam combination have been proposed in the literature. In this article, we compare these beam combiners by rigorously simulating the imaging process of a weak stellar source, taking into account the photon arrival statistics, an imperfect detection process and the image reconstruction from the recorded data. The results are presented as the to be expected reconstruction error in the luminous intensity distribution function of a wide-field stellar source versus the provided amount of photons. Using these results, the optimum design of the combination beam combiner and detector array can be identified. [DOI: 10.2971/jeos.2006.06020]

Keywords: Coherence imaging, partial coherence in imaging, phased-array imaging systems, telescopes, astronomical optics, space optics

\section{Introduction}

With successful optical stellar interferometers in operation on Earth, stellar interferometry has proven to be a valuable observation technique [1]-[3]. Two or more telescopes observe the same source and, by coherent combination of the collected radiation, information is gained about the source with an angular resolution directly related to the separation of these telescopes, rather than the diameter of a single telescope.

In the near future, space-based stellar interferometers will be constructed to detect signals from possible Earth-like planets around nearby stars. The Darwin mission of the European Space Agency (ESA) aims at such a constellation of telescopes [4]. Another space-based interferometer will be the Terrestrial Planet Finder Interferometer or TPF-I by NASA. The value of these missions can be greatly expanded by the addition of an imaging mode, allowing aperture synthesis imaging next to the nulling task [5]. With baselines in the case of Darwin in the order of hundreds of meters, these imaging observations can provide information on young stellar objects with unprecedented accuracy. This may answer questions about the origin of galaxies and planetary systems.

Optical stellar interferometry is characterized by the recording of intensity interference patterns from a stellar source. In general, the interference pattern has a limited extent due to the passband of optical frequencies that are recorded. With- out fringes, the combination is incoherent and besides a larger light collecting area, this is not improving the imaging. Unlike the practice in radio astronomy, the selection of a single frequency is impossible and noise-free amplification of collected electro-magnetic fields at each telescope does not exist. Another difference is the field-of-view (FOV). An optical telescope has a FOV of many times the size of the diffractionlimited spot, or point spread function (PSF). Radio interferometry relies on imaging within a single PSF and mosaicing a field together. Conventional imaging with a single optical telescope can provide a picture, e.g. from a CCD-camera, of a much wider field. In optical interferometry, the goal is to image a field possibly as large as that of a single telescope having the resolution of the interferometer array.

It is our goal to treat all interferometers, or rather the beam combiners that identify them, as optical imagers. This allows coverage of polychromatic observations as well as wide-field interferometry in a straightforward manner. As detailed in a previous publication, the effects addressed by partially coherent imaging are covered [6]. The occurrence of discontinuous optical surfaces or complicated beam relay optics-as these occur in some of the beam combiner concepts-poses no limitation to our approach, whereas a treatment with the theory for partially coherent imaging (e.g. using Hopkins' formulation [7]) - the usual foundation for describing interferometric 
imaging-would require an intensive mathematical formulation. However, such an approach is possible [8] and should be considered when time consumption by the presented purely numerical approach poses a limiting factor in the design process of an interferometer.

The theory and simulations presented in this paper assume a space-based array of telescopes operating at the diffraction limit. A study of the occurrence of aberrations and the applicability to Earth-based interferometers can be found in the literature [8]. The analysis and simulations described in this article have a limited complexity for ease of presentation. A two-element interferometer is considered, delivering high angular resolution in only one direction. The simulated stellar source has a complicated and fine spatial structure. The assumed and collected spectrum is broad banded, but constant over the source.

\section{M O D E LIN G}

This section will describe the one-dimensional model used in the simulation. Our analysis starts with the observation that a stellar source can be considered as an incoherent collection of point sources, originating from a range of directions $\theta_{\text {in }}$. Any optical imager-either monolithic or interferometricdelivers an intensity response at the detector as a function of $\theta_{\text {out }}$. Interferometers are equipped with a delay line, causing the intensity response to be a function of the delay $d$ as well. Figure 1 shows four sketches of common beam combiner optics for stellar interferometers.
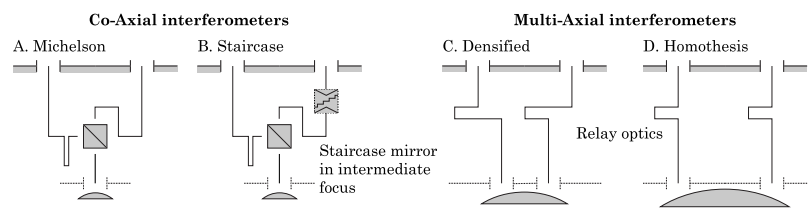

FIC. 1 Sketches of four stellar interferometers as described in the literature. Note that Michelson's experiments on stellar interferometry were performed with a configuration similar to sketch $C$. The label Michelson as used here and in the literature refers to the co-axial Michelson-Morley interferometer. Configuration $D$ is commonly addressed as a Fizeau interferometer, but it should be noted that Fizeau experimented with a masked aperture; no beam relay optics were needed.

Table 1 provides the parameters that characterize these four beam combiners.

\begin{tabular}{lcccc}
\hline & Homothetic & \multicolumn{2}{c}{ DensifiedMichelsonStaircase } \\
\hline Axiality & multi & multi & co-axial & co-axial \\
Recombination & $B_{\text {out }}=B_{\text {in }}^{(k)} / M B_{\text {out }}=4 D$ & $B_{\text {out }}=0$ & $B_{\text {out }}=0$ \\
Magnification & $M=1$ & $M=1$ & $M=1$ & $M=1$ \\
Fringe encoding & $\theta_{\text {out }}$ & $\theta_{\text {out }} d$ & $d$ & $d$ \\
\hline
\end{tabular}

TABLE 1 Characterization of the beam combiner optics in the interferometers under consideration.
The item fringe encoding refers to whether the fringed intensity distribution on the detector is to be measured as a function of delay $d$ and/or as a spatial variation across the detector in direction $\theta_{\text {out }}$. The fringed intensity output delivered by the beam combiners can be described by a single analytical expression, needing only a small modification to deal with the discontinuity present in case B, where a staircase-shaped mirror is present. As indicated in Figure 2, the general Fraunhofer (far-field) intensity output from a two-element interferometer caused by a single infinitely small point source is given by

$$
\begin{aligned}
& I_{k}\left(\theta_{\text {out }}, d\right)=4 D^{2} \operatorname{sinc}^{2}\left[\frac{D \pi}{M \lambda}\left(\theta_{\text {out }}-M \theta_{\text {in }}\right)\right] . \\
& \cos ^{2}\left[\frac{\pi}{\lambda}\left(B_{\text {out }} \theta_{\text {out }}-B_{\text {in }}^{(k)} \theta_{\text {in }}+d\right)\right] .
\end{aligned}
$$

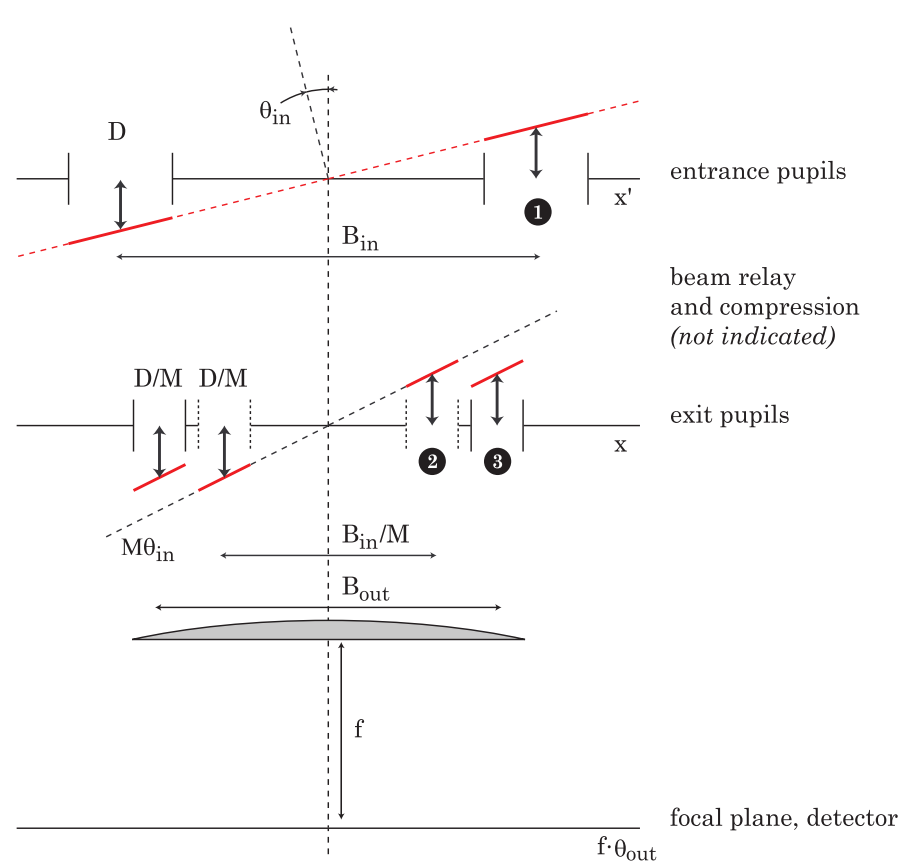

FIG. 2 This illustration indicates the coordinates used in the derivation of the pointsource response functions for all types of beam combination (any $B_{\text {out }} \geq 0$ ), when a general beam compression by a factor $M$ is applied in each arm. The diffraction integral is constructed symmetrically, leading to a compact expression. The phase differences at the collection plane $x^{\prime}$ are indicated with black arrows at the locations numbered 1,2 and 3. After beam compression, the phase slope in an aperture in the plane $x$ is proportional to $-x M \theta_{\text {in }}$. The arrow at location 2 indicates that for proper exit pupil placement at a separation $B_{\text {in }}^{(k)} / M$ the light paths of a masked aperture are followed, since the wavefronts in the apertures are part of a single wavefront. Cenerally, see indicator 3 , the aperture separation after beam relay is $B_{\text {out }}$, which is zero for co-axial beam combination. As a result, the wavefronts in the exit apertures, see indicator 3 , are parallel to the dotted line $-x M \theta_{\text {in }}$ but have an off-set. As a result, a diffraction integral can be constructed, in which the phases proportional to $\left[-x M \theta_{\text {in }} \pm \frac{1}{2}\left(B_{\text {in }}^{(k)} / M-B_{\text {out }}\right) M \theta_{\text {in }}\right]$ occur.

Here, the $k^{\text {th }}$ collection baseline $B_{\text {in }}^{(k)}$ and telescope diameter $D$ are parameters of the array, independent from the beam combiner. The response is further governed by the magnification or beam compression $M$, the recombination baseline $B_{\text {out }}$ and the wavelength $\lambda$.

A previous publication [6] describes the properties of the $I_{k}\left(\theta_{\text {out }}, d\right)$ response in Eq. (1) for a two-element interferometer in the cases of Homothetic imaging [9], Densified pupil 
imaging [10], co-axial Michelson imaging [11] and Staircase imaging [12]. The collection of point sources in the FOV is expressed as a luminous intensity function $L\left(\theta_{\text {in }}\right)$, causing a sum of intensity patterns on the detector. Performed (and weighted) for many wavelenghts $\lambda$, polychromatic fringes with limited extent arise. We state again that the function $L\left(\theta_{\text {in }}\right)$ is taken wavelength-independent in these simulations. The theoretical intensity pattern, however, will only be detected as such in abundance of photons. A noise model will be implemented to describe the detected signal as a function of the amount of photons arriving from the stellar source.

Optical interferometers observe weak stellar sources and are therefore said to operate in the photon-starved regime. To adequately simulate low-light level observations, a noise model considering the statistics of photon arrival is necessary. Moreover, since the detected data are the start of an inversion process, these data should represent realistic noisy recordings since the inversion and hence the final image formation shall be limited by the noise level in the recordings. Therefore, the noisy read-out process of an infrared detector array is also simulated.

The analytical expressions for the intensity detector response, derived in Eq. (1), predict the 'image' that the detector array will observe. However, this pattern will only be observed for very bright sources, when unlimited amounts of photons are available. The response of a weak source, such as the astronomical objects of interest, is better expressed by taking into account the arrival of photons from the object, at a certain location and at certain time intervals [13]. Figure 3 illustrates this for an increasing number of arrival events.

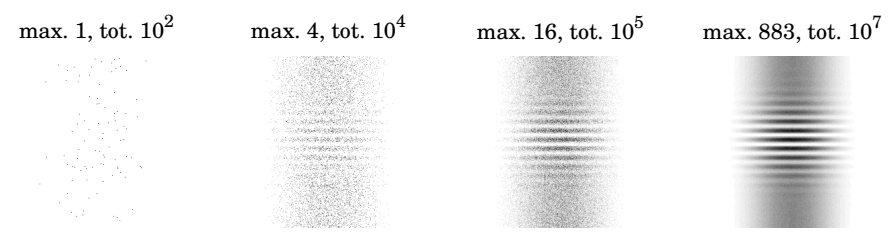

FIG. 3 A polychromatic fringe pattern $I\left(\theta_{\text {out }}, d\right)$ out of a co-axial two-aperture interferometer. The images from left to right show observations of the theoretically predicted fringe pattern, moving from a photon-starved to a photon-rich regime. The maximum amount of photons per bin is indicated (max.) as well as the provided total amount of photons (tot.). These examples exhibit zero detector noise.

The analytical intensity distribution $I\left(\theta_{\text {out }}, d\right)$ is now seen as a likelihood distribution over space and time, where time in this case (co-axial combination) is the applied delay $d$. Timedependency stems from the shot-noise, which is the arrival probability of a photon. This probability follows Poissonian statistics. The analytical intensity response defines the average fluxes in all bins ('tiles' in $\left(\theta_{\text {out }}, d\right)$-space), being pixels and delay settings. For each bin, with its average flux, the number of photons per readout (after an integration time $t_{\text {int }}$ ) is then discretized, in such a way that the total number of photons is a specific desired amount $N_{\text {tot }}$, that can be chosen to match the integration time $t_{\text {int }}$ and the magnitude $m_{v}$ of the stellar object. The simulated amount of photons per bin (pixel and delay) is then returned, based on the calculated average amount, by an implementation of the Poisson statistics, as described in $\mathrm{Nu}$ merical Recipes [14]. A few examples are depicted in Figure 3.

Thermal radiation of other origin than the stellar object, for example warm telescope optics, cause the presence of background counts, which are also Poisson distributed. Readout noise (the electronics register non-existing photons) further contributes to the degradation of the acquired images. This noise is characterized as a Gaussian random process. Since the radiation from the object and the background are indistinguishable and follow the same statistics, there is no need to account for this noise in the simulations, other than taking a source $L\left(\theta_{\text {in }}\right)$ that incorporates a certain background level. For the readout noise, an actual camera should be considered, in order to arrive at a sensible mean value for the amount of electrons (photon arrival events can also be expressed in electrons) added to the detected count, and a proper standard deviation. As an example, the IRAC camera for the Spitzer Space Telescope is considered $[15,16]$. The measured total readout noise can be taken to have a mean $\mu=15$ electrons $\left(e^{-}\right)$and a variance $\sigma^{2}=8 e^{-}$. Note that these values are depending on gain settings and numerous device-dependent parameters. The mean value is usually subtracted from the data, which may result in negative values or counts in the detected signals.

It should be noted that in interferometry, 'background' signal is also generated by the source itself. In a polychromatic situation, the 'incoherent' tails of two fringe packets from two closely separated point sources may cause the summed intensity pattern to have a large non-modulating component. For the detector signal, only the modulating part holds the information for high resolution. The background hence contains 'non-information', but the classical definition of background would be 'information from elsewhere than the source'.

\subsection{Simulations}

A set of simulations is performed in order to predict the imaging performance of the Darwin array. The array under consideration is a two-element configuration, generating a onedimensional, high-resolution estimate of the luminous intensity distribution on the sky $\hat{L}\left(\theta_{\text {in }}\right)$. The simulations are performed at the ultimate values allowed by the software and the computer. Since a linear system of equations lies at the basis of the simulation process, large values of the field-of-view (FOV) and ultimate angular resolution generate matrices that reach the storage capacity of the internal memory of the computer on which the simulations are run, in our case a desktop Pentium IV with $500 \mathrm{MB}$ internal memory. For that reason, the following simulations do not reach the requirements for the DARWIN imaging mission, listed in the literature [4]-[8]; the achieved angular resolution is $71 \mathrm{nrad}$, whereas the requirement was 0.005 " or 24 nrad. For the comparison this is completely irrelevant, since it relates physically to the locations of the collecting telescopes, which are the same for the four imaging methods.

The original source function or luminous intensity distribution $L\left(\theta_{\text {in }}\right)$ is taken to be a cross-section of the object $\mathrm{HH} 47$. 
In Figure 4 , the function $L\left(\theta_{\text {in }}\right)$ is depicted, together with the point-spread function (PSF) of a single telescope.

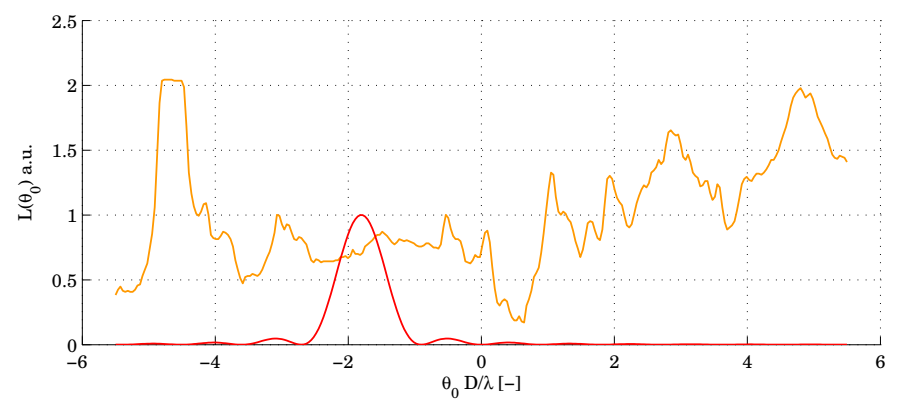

FIG. 4 The function $L\left(\theta_{\text {in }}\right)$ is a normalized cross-section through an image of the object HH47 (gray line). Its angular extent is chosen to span 11 incoherent PSFs; one single PSF is indicated with the black line. The details to be reconstructed in $\hat{L}\left(\theta_{\text {in }}\right)$ are much smaller than the incoherent PSF or the resolution of a single telescope. The vertical axis is of arbitrary units (a.u.).

The source function is chosen to span approximately 11 PSFs. The characteristics of the different beam combiners are listed in Table 1. The magnification or beam compression $M=1$ is chosen for simplicity. The detector array has the same angular span as the source function. The telescope diameter is $D=3.5 \mathrm{~m}$. The baseline lengths $B_{\text {in }}^{(k)}$ at which snapshots are taken, are then given as

$$
B_{\text {in }}^{(k)}= \begin{cases}2 D & k=1 \\ 4(k-1) D & k=2,3, \ldots, 9\end{cases}
$$

The baseline length differences of $4 D$ seem to be large, but with an observational bandwidth for which $6 \mu \mathrm{m} \leq \lambda_{c} \leq$ $10 \mu \mathrm{m}$, this set of baselines leads to a spatial frequency coverage with no gaps up to the desired ultimate spatial frequency, expressed as angular wavenumber, given by

$$
\frac{B_{\text {in, } \max }}{\lambda_{c}}=\frac{112}{8 \cdot 10^{-6}}=1.4 \cdot 10^{7}\left[\mathrm{rad}^{-1}\right] \text {. }
$$

The frequency coverage is illustrated in Figure 5.

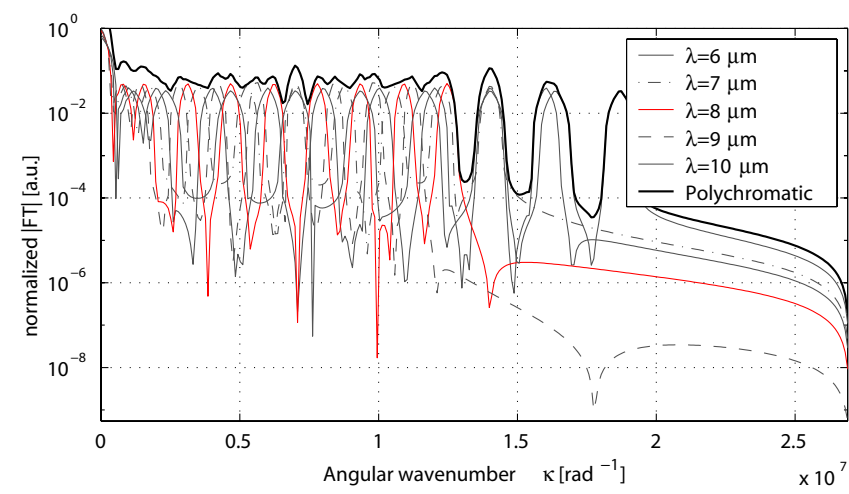

FIC. 5 To check the coverage of spatial frequencies, monochromatic homothetic PSFs are calculated for $\lambda=6,7,8,9,10 \mu \mathrm{m}$ and the baseline lenghts $B_{\mathrm{in}}^{(k)}$ of expression Eq. (2). Summing the intensity PSFs over all $k$, the Fourier Transform (FT) of these PSFs can be taken and the normalized absolute values are plotted. Per trace, the spacing of peaks corresponds to the $4 D$ gaps in baseline lengths. The FT of the sum of all PSFs is plotted as a thick black line and represents the spatial frequency coverage for the complete sets of baseline lengths as well as wavelengths.

The 11 PSFs wide source function is discretized on a 333points equidistant grid. This leaves 33 resolution elements per PSF, matching to the ratio $B_{\text {in, } \max } / D=32$, since the separation of the telescopes dictates the ultimate resolution, not the gridsize for the reconstruction of $\hat{L}\left(\theta_{\text {in }}\right)$. Therefore, all features in the function $L\left(\theta_{\text {in }}\right)$ are recoverable, leading to reconstruction error $\epsilon$-values that are only limited by shot-noise. A full description of the estimation process of $\hat{L}\left(\theta_{\text {in }}\right)$ is described in an earlier publication [6].

Table 2 provides a list of the detector discretizations, needed for the four different imagers, in order to cover the FOV and reach the same angular frequency coverage.

\begin{tabular}{lcccc}
\hline \multicolumn{4}{c}{ HomotheticDensifiedMichelsonStaircase } \\
\hline Detector size & $F O V$ & $F O V$ & $F O V$ & $F O V$ \\
Pixel size & $\frac{p_{\theta}\left(B_{\text {in, } \max }\right)}{4}$ & $\frac{p_{\theta}\left(B_{\text {out }}\right)}{4}$ & $\frac{p_{\theta}(D)}{2}$ & $\frac{p_{\theta}(D)}{2}$ \\
Number of pixels & 1562 & 101 & 20 & 20 \\
Scanning range & - & $\Lambda$ & $\beta$ & $2 l_{\text {coh }}$ \\
Delay stepsize & - & $10 \lambda_{c}$ & $\lambda_{c} / 4$ & $\lambda_{c} / 4$ \\
Number of steps & 1 & 36 & 1561 & 25 \\
Bins total & 1562 & 3636 & 31220 & 500 \\
\hline
\end{tabular}

TABLE 2 Discretization of the $\left(\theta_{\text {out }}, d\right)$ detection space for the simulations. Here, $\Lambda=-187 \lambda_{c} \ldots+187 \lambda_{c}$ and $\beta=2 B_{\text {in, } \max } \theta_{\text {in }}^{(\max )}$. The angular fringe period in focus $p_{\theta}$ is derived from the general intensity response function and equals $p_{\theta}\left(B_{\text {out }}\right)=\lambda_{c} / B_{\text {out }}$. The discretization ensures that all high-frequent spatial information of the stellar source is presented through fringes in $\left(\theta_{\text {out }}, d\right)$-space and can be detected.

For the methods other than Densified, the discretization is straightforward. The necessary range of delay steps for the Densified case to have coherent information from all regions of the sky, is derived from the modulation part of the intensity point-source response for a general interferometer, given as

$$
\begin{gathered}
\cos \left[\frac{\pi B_{\text {out }}}{\lambda}\left(\theta_{\text {out }}-\left[\frac{B_{\text {in }}^{(k)}}{B_{\text {out }}} \theta_{\text {in }}\right]+\frac{d}{B_{\text {out }}}\right)\right]=1, \\
\text { for } \theta_{\text {in }}=\theta_{\text {in }}^{(\max )}
\end{gathered}
$$

The expression of Eq. (4) states that a bright fringe is necessary at the boundary of the field-of-view. Since the observation is polychromatic, this requirement is reduced to finding the delay $d$, for which the central fringe appears in the center of the diffraction envelope. This center is located at $\theta_{\text {out }}=\theta_{\text {in }}^{(\max )}$, so that Eq. (4) now reduces to

$$
d=B_{\text {out }} \theta_{\text {in }}\left(1-\frac{B_{\text {in }}^{(k)}}{B_{\text {out }}}\right) .
$$

With the given FOV of 11 PSFs wide, a combination baseline length of $B_{\text {out }}=7 \mathrm{~m}$ and a maximum collection baseline length of $B_{\text {in }}=112 \mathrm{~m}$, Eq. (4) yields a maximum delay $d_{\max }=1.5 \mathrm{~mm}$, corresponding to $187 \cdot \lambda_{c}$. The amount of steps 
to go through this delay-range is then chosen such that for every direction $\theta_{\text {in, }}$ the corresponding intensity PSF shows an envelope with clearly visible fringes. This amount is related to the coherence length and can be found by inspection of the calculated PSFs. Increasing the amount of steps leads eventually to a PSF with a central fringe in it, for every sky coordinate

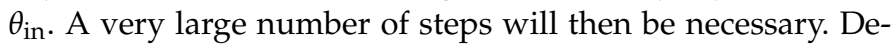
creasing the amount of steps leads to regions of the sky for which the PSF hardly shows fringes, since only for the values of $\theta_{\text {in }}$, matching to the delays $d$ according to Eq. (5) the PSF will show a central fringe in the center of the PSF. The 11 PSFs wide sky is adequately covered with 36 steps of $d$, spanning the $-1.5 \ldots+1.5 \mathrm{~mm}$ range.

For the Staircase method, the number of stairs was chosen to be equal to the number of pixels. The stair heights $h_{0}$ are optimized for each baseline length. The stair height $h_{0}$ might in practice also be chosen to be fixed when an actuatable staircase-shaped mirror is not feasible. The reduction of the delay range in which fringed information is present still applies, but is not optimal [8]. As a result of variable stair heights, all fringes are present within a very short $d$-range. The ultimate delay $d$ at which the central fringe can be found, is a function of the stair width and the baseline length-and is in the $h_{0}$-optimized case equal to the stair-height.

\subsection{Quantitative comparison}

After the simulation process the estimate of the luminous intensity distribution can be compared with the known luminous intensity distribution. For $N$ resolution elements on the sky, the normalized error in the estimate or the difference norm $\epsilon$ is calculated:

$$
\epsilon=\frac{1}{N} \sqrt{\sum_{i}\left|s_{i}-\hat{s}_{i}\right|^{2}} .
$$

Where $s_{i}$ is the intensity level of one resolution element in the source and $\hat{s}_{i}$ is the corresponding element in the estimate. For the simulations, a non-existing stellar source is taken. The source luminous intensity function $L\left(\theta_{\text {in }}\right)$ has a mean amplitude equal to unity; a variable and realistic photon flux is realized by adjusting the discretization of the measurement signals, rather than adjusting the source function. Therefore, the source function can be normalized so that it has a mean value of 1 and the estimate will also have an approximate mean value of 1 . If then the comparison of an estimate to the original according to Eq. (6) yields an $\epsilon=0.01$, this means that the dynamic range in the reconstruction is $1: 100$.

Another reason to support the use of $1 / \epsilon$ as measure for $d y-$ namic range is the following. The dynamic range is the ratio of the intensity resolution in the image, compared to the maximum value. Since the power in the original luminous intensity function $L\left(\theta_{\text {in }}\right)$ is normalized to 1 , the amplitude in the Fourier spectrum $\mathcal{F}\left[L\left(\theta_{\text {in }}\right)\right](k)$ for $k=0$ is 1 . And since $L\left(\theta_{\text {in }}\right)$ is positive, all other values in $\mathcal{F}\left[L\left(\theta_{\text {in }}\right)\right](k)$ are smaller. And indeed, a low resolution version of $L\left(\theta_{\text {in }}\right)$ will show a rough envelope function resembling $L\left(\theta_{\text {in }}\right)$, with a periodic cosinelike oscillation around that envelope. The amplitude of that periodic function is related to the largest Fourier amplitude that was left out. Leaving out higher spatial frequencies either because a limited $B_{\text {in,max }}$ is taken or because too much filtering is needed in case of a photon-starved observation, results in a higher value of $\epsilon$. And indeed, following the definition of dynamic range, short baseline observations or observations with a lot of (photon-)noise in it, will result in a low dynamic range $1 / \epsilon$.

\section{R E S U L T S}

\subsection{Comparison of the transfer matrices}

The transfer matrices for the four methods can be composed and analyzed with Singular Value Decomposition [6]-[8]. The use of the SVD is presented in these publications. It decomposes the imaging process into a set of nearly orthogonal basis vectors $\mathbf{v}_{i}$, of which an estimate is constructed. These basis vectors directly relate to physical parameters in the imaging process. The series of singular values $\sigma_{i}$ are plotted in Figure 6, where each series is normalized by its largest singular value, $\sigma_{1}$.

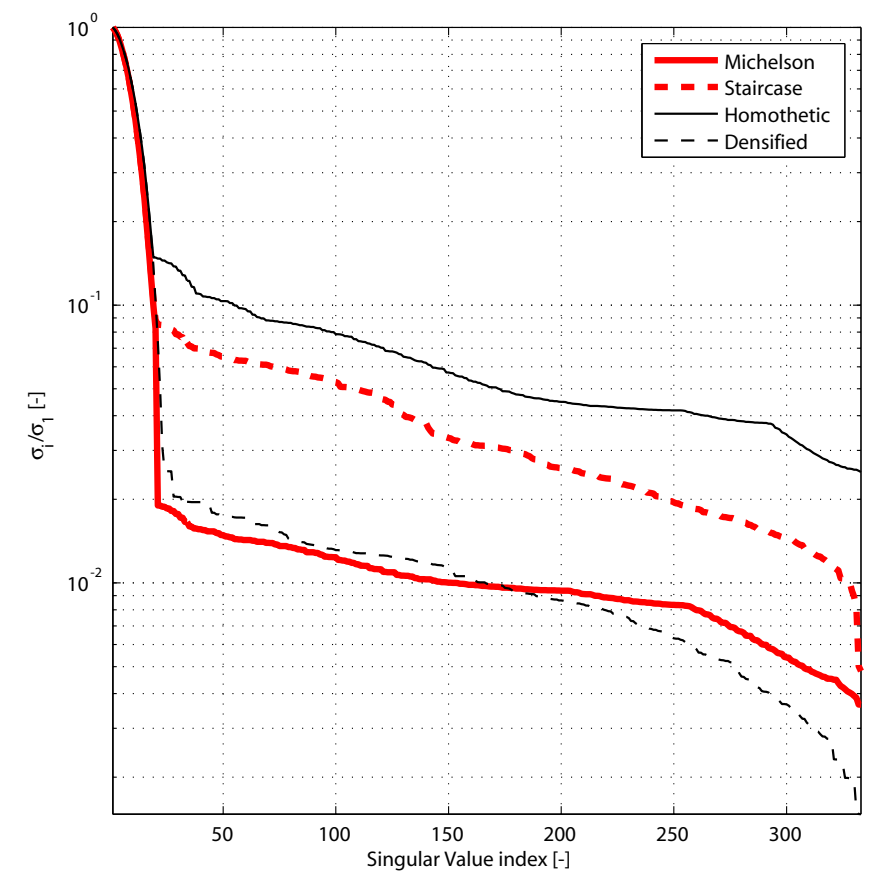

FIG. 6 The singular values $\sigma_{i}$ are normalized with respect to the largest singular value $\sigma_{1}$. This allows comparison of the responsiveness of the imaging method with respect to spatial frequencies in the observed luminous intensity function $L\left(\theta_{\text {in }}\right)$. The graphs show an incoherent imaging regime, for indices $i \leq 20$, and a coherent imaging regime, for indices $20<i \leq 333$. There is no unsampled regime, because of the very long baseline lengths.

Inspection of the plots provides information as described, in detail, below.

Up to index $i=20$, the singular values drop heavily. By inspecting the singular vectors $\mathbf{v}_{i}$ for these indices one concludes that these modes correspond to the incoherent regime. With a resolution, governed by telescope diameter $D$, the flux is addressed to the 11 PSFs wide sky. Apparently, a set of 
$\simeq 11 \times 2$ SVD modes is sufficient for this task. The co-axial imagers have 20 pixels on the detector, but the image-plane methods have much more. In the coherent regime, for which $20<i \leq 333$, the average level of the slowly decreasing lines is a measure for the amount of coherent information in the detection signals. Homothesis and Staircase both have fringe information in all regions of every snapshot they record, whereas Densified and Michelson record large amounts of incoherent intensity data per snapshot. This is a direct result of the non-zero bandwidth in use and the resulting finite coherence length. However, not only the average level of the curves is related to quality. The 'flatness' of the traces is a measure for the coverage of the field at all spatial frequencies. The flatness of Homothesis and Michelson is better than that of Staircase and Densified. Apparently, for a certain spatial frequency, not every direction $\theta_{\text {in }}$ is represented with equal strength. In other words, these two non-classical methods have a fieldvariant response, changing with the spatial frequency. However, this does not mean that all information of $L\left(\theta_{\text {in }}\right)$ cannot be restored. For the Staircase method, an analysis of the slight inefficiency as compared to co-axial Michelson is presented in Appendix A. For the Densified method, the reason for the slight inefficiency lies in the fact that only a limited set of values of $d$ is taken per baseline configuration, and that the fringe packet moves out of the diffraction envelope.

The analysis of the transfer matrices by inspection of their singular values, can be done before starting simulation runs for each method. Facts such as complete coverage of field at all spatial frequencies, and a correct number of singular values related to the incoherent image, can be checked. The simulations can then be started with transfer matrices that match the desired imaging parameters, such as field and resolution. But even without simulations, the curves in Figure 6 already predict which imaging method will perform best. The method that demonstrates in its $\sigma_{i} / \sigma_{1}$-trace

a limited drop of magnitude between the incoherent and the coherent regime,

an as limited as possible decrease of magnitudes in the coherent regime, and

a coherent regime continuing until index $i$, where $i$ is equal to the number of resolution elements in the function $L\left(\theta_{\text {in }}\right)$,

is stated to be the best performing imaging method. The numerical simulations, incorporating detector-noise and shotnoise, should prove this statement.

\subsection{Comparison of simulated ob- servations}

Figures 7 and 8 represent the results of 100 simulations.

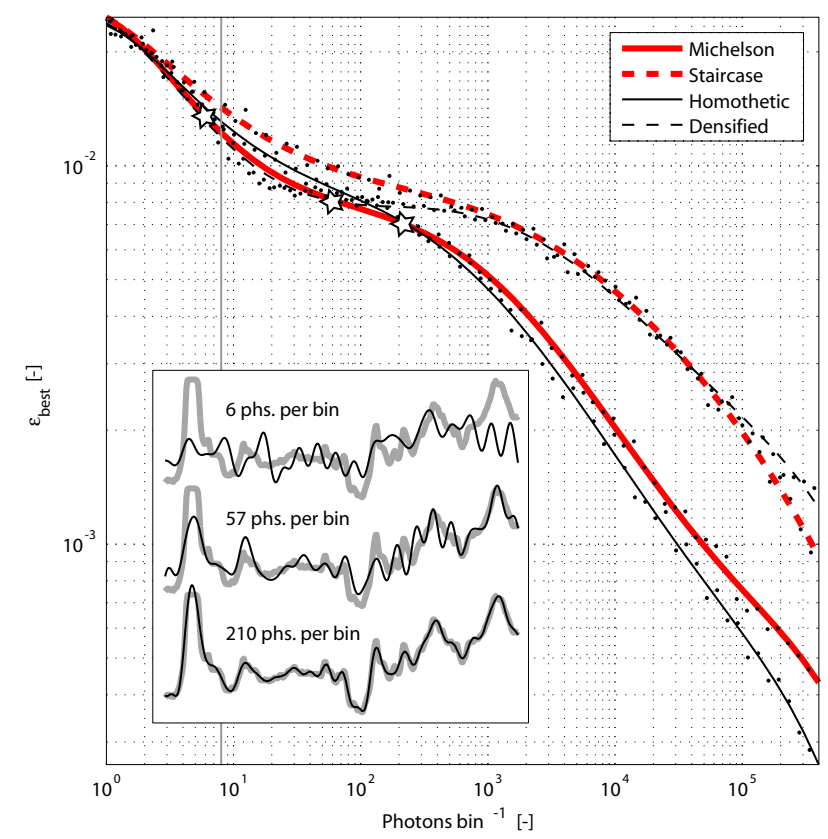

FIG. 7 The normalized reconstruction error $\epsilon$ is expressed as a function of the number of photons per bin. Above the indicated detector noise level of 8 photons per bin, the curves indicate the efficiency of the imaging methods. Polynomials are fit through the data, for displaying purposes only. In the inserts, three homothetic reconstructions are presented, obtained with respectively 6,57 and 210 photons per bin (see position of the 'stars'). At the very low light levels, a nearly random reconstruction at the proper average level results in a misfit of $\epsilon=10^{-1.67}$. Above the level of 210 photons per bin, only the very small features in $L\left(\theta_{\text {in }}\right)$ are still to be recovered. For this reconstruction, the misfit is $\epsilon=10^{-2.22}$ and the dynamic range is $1 / \epsilon=167$. The less-steep region around $\epsilon=10^{-2}$ indicates the transition from incoherent to coherent imaging

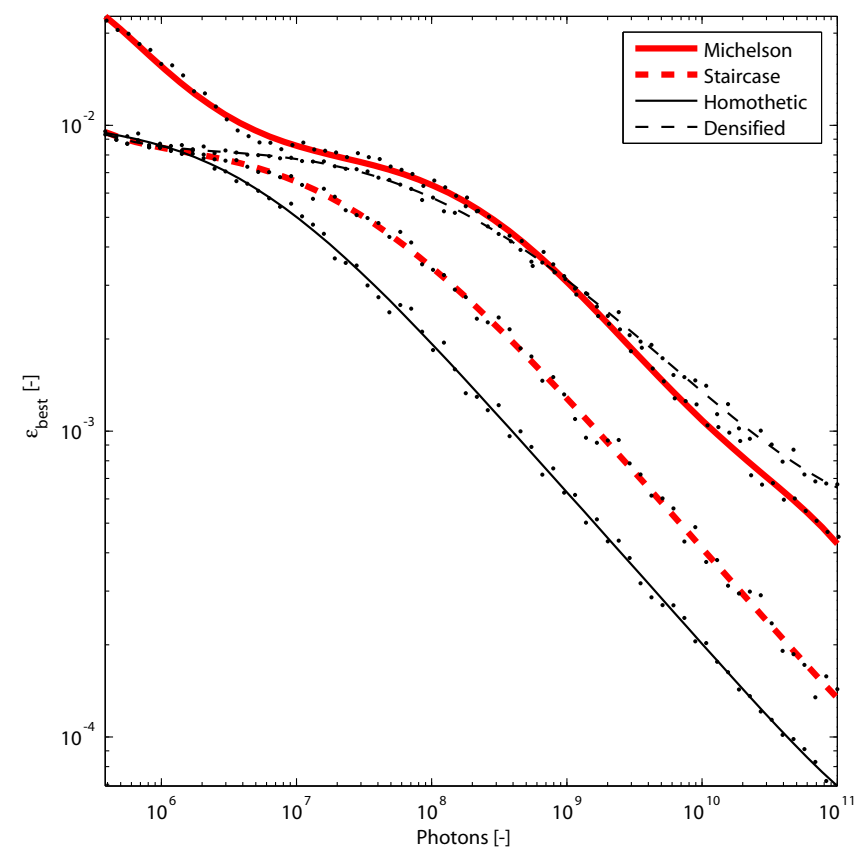

FIG. 8 The normalized reconstruction error $\epsilon$ versus the total amount of received photons for the complete observation of $L\left(\theta_{\text {in }}\right)$. The huge amount of bins in the Michelson case causes that curve to deviate from the others. Polynomials are fit through the data, for displaying purposes only. 
The four imaging methods are applied to the same source $L\left(\theta_{\text {in }}\right)$, from which an increasing number of photons is received. Figure 7 displays the imaging efficiency, expressed as the reconstruction error $\epsilon$, for the best reconstruction possible, versus the number of photons per bin (being a tile in $\left(\theta_{\text {out }}, d\right)$ space). Detector noise is again 8 photons per bin. Above this amount of photons per bin, all imaging methods demonstrate a drop in reconstruction error; this marks the point where significant imaging starts, be it that the reconstructions will have a resolution comparable to that of incoherent or single-dish imaging. The region around $\epsilon=10^{-2}$ demonstrates the transition to coherent imaging. Sufficient photons available cause the fringes in the detected signal to be distincted from the incoherent envelope function. For more than $10^{3}$ photons per bin, the interferometric imaging efficiency related to the four beam combination schemes comes visible. The methods Homothesis and Michelson-the two classical methods-have a comparable efficiency and perform better in terms of efficiency than the methods Densified and Staircase. The reasons for this lie in the large amount of incoherent information and the use of multiplexing, respectively.

The amount of incoherent information in the detected signals is larger for Densified as compared to Homothesis. Because of field-dependent internal path length compensation, the fringe packet 'drifts' out of the incoherent envelope function for Densified. As explained, this is overcome by imaging the sky at the same baseline configuration several times with changing the delay $d$. Each recording contains fringed data for one certain region of the sky, but adds incoherent data for the other regions of the sky. The total collected information per baseline length contains a large amount of incoherent data, resulting in a low efficiency. A likewise problem related to the loss of fringes because of the finite coherence length is found in the co-axial imagers. The Staircase imager compensates the external field-dependent path length in a discrete way, effectively resulting in fringed response signals for the entire field, within a reduced range of delay $d$. For this case, however, the lower efficiency lies not in the fact that more incoherent information is recorded; as much as all recorded information will be fringed. The elementary response signals $I_{k}\left(\theta_{\text {out }}, d\right)$ consist of an Airy-like envelope in direction $\theta_{\text {out }}$ and a modulation signal in direction $d$ constituted from a constant value and a polychromatic fringe function of a length related to the coherence length. The response of the sky can be considered to be multiplexed in $d$-direction, because of the staircase-shaped fielddependent compensation. The width of the response functions in direction $\theta_{\text {out }}$, being at least two pixels on the detector, together with the fact that no positivity constraint is present in the estimation process of a reconstruction of the sky, cause a non-uniqueness of detector response ensembles to intensity distributions on the sky. Hence, a lower efficiency in terms of reconstruction error versus photons per bin results.

Figure 8 finally, shows the performance of the four imaging methods as a function of observation time. Whether the best reconstruction is sought for a given observation time, or the shortest observation time is sought provided a minimum reconstruction quality, the outcome will be the same. The curves essentially are horizontally shifted versions of those in Figure 7 . Here one can see that the best imaging method is
Homothesis, followed by the Staircase method. For a given amount of photons or observation time, these beam combiners for an equal set of telescopes provide reconstructions of the wide-field source containing the most features.

\section{DISCUSSION AND C ONCLU- $S I O N S$}

Based on the equal treatment of the signals each interferometer provides and the resulting figures of reconstruction quality versus the number of photons received, we conclude that Homothesis is the superior imaging method for wide-field, high angular resolution images. Not surprisingly, it is this method that resembles most the ideal situation of a very large telescope with an aperture mask in front of it. However, the construction and operation of a homothetic imaging array are no trivial issues, as described in the literature [17, 18]. The Staircase method achieves storage of high resolution information from the sky into a minimal number of detector binswith only 500 bins, it has by far the smallest amount. Although the reconstruction process suffers slightly from this data-compaction by multiplexing, the Staircase method performs very well in the sense that it is fast, or, in other words, that it needs less photons to reconstruct an image than Michelson or Densified. As in the case of Homothesis, there are practical issues to overcome. A staircase-shaped mirror with a certain number of aligned facets can be produced [12]. But then, either an actuatable version with tuneable stair-height has to be designed, or a system should be installed that can replace a rigid staircase mirror for each of the $k$ observations with baseline lengths $B_{\text {in }}^{(k)}$, in order to match the stair height $h_{0}$ to the baseline length.

Considering the merit of the analysis of the transfer matrices, the conclusion can be drawn that inspection of the singular values is a quick and accurate means to predict the imaging performance of an interferometer. The superiority of Homothesis, followed by the Staircase method, seen in the singular value plot is in accordance with the final simulation results where the simulated detection process and final reconstruction identify the same two methods to perform best.

\section{A APPENDIX: INEFFICIENCY OF STAIRCASE RESPONSE FUNC- TIO N S}

Besides the loss of light due to the presence of staircase discontinuities or edges in the intermediate focal plane, there is also a signal processing-related 'loss' connected to the Staircase method. As described, the production of estimates $\hat{L}\left(\theta_{\text {in }}\right)$ is, in the case of Staircase imaging, slightly less efficient than in the case of Michelson imaging. Despite the fact that the Staircase compacts the fringed measurement signals, it needs more photons per bin to obtain an estimate of equal fit to the original. This inefficiency is illustrated in Figure 7, where the Staircase curve is far above the Michelson curve, indicating a worse reconstruction at an equal amount of photons per bin. The inefficiency can be explained by the nature of the fringed 
signal. In the case of Staircase imaging, there is no unique relation between the location and intensity of a point source on the sky and the resulting single-point response function.

Following the staircase-induced staggered line in $\left(\theta_{\text {out }}, d\right)$ space for the origin location of the envelope and the fringes, one can think of situations where the integrated response signal in a pixel, as function of $d$, is the same, while a different $L\left(\theta_{\text {in }}\right)$ caused these responses. Figure 9 illustrates such a situation, based on the fact that negative contributions $\left(L\left(\theta_{\text {in }}\right)<0\right)$ to a response are, in principle, allowed.

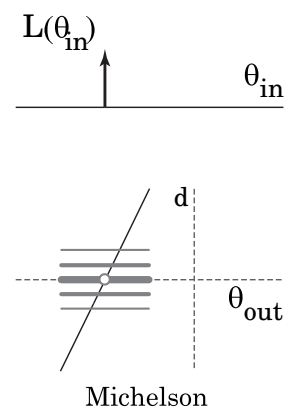

(a)

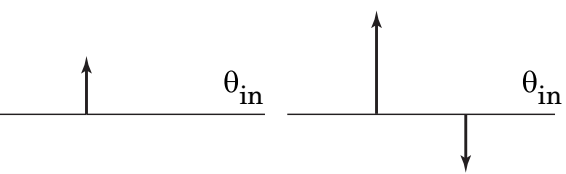

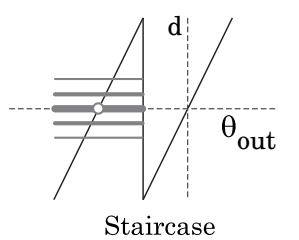

(b)

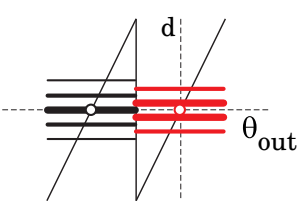

Staircase

(c)
FIG. 9 Three elementary source functions $L\left(\theta_{\text {in }}\right)$ are depicted. The detector responses in $\left(\theta_{\text {out }}, d\right)$-space are given, at a certain baseline $B_{\text {in }}^{(k)}$. The steepness of the diagonal line relates to $B_{\text {in }}^{(k)}$; the center of a point source response from direction $\theta_{\text {in }}$ ends up centered around $\left(\theta_{\text {out }}, d\right)=\left(M \theta_{\text {in }}, B_{\text {in }}^{(k)} \theta_{\text {in }}\right)$. The response in the Michelson case (a) uniquely matches to the given source function. Since in the case of Staircase imaging the fringe data is compacted, the two sketched responses in (b) and (c) provide after integration over a pixel the same intensity signal $I_{j k}(d)$, but a totally different-yet allowed-source function $L\left(\theta_{\text {in }}\right)$.

In the sketched situation, the combination of a large and positive response with a small and negative response yields the same measurement as the combination of a single, smaller, positive response. Due to the fact that different source functions $L\left(\theta_{\text {in }}\right)$ can lead to the same or nearly the same measurement, the Staircase method will need more information to be able to give a correct estimate of the sky. Therefore, given the same set of baseline lengths as the Michelson case, it will perform worse, as is indicated by the singular values in Figure 6, where the normalized singular values of the Staircase imager show a steeper drop-off, and is confirmed by the numerical simulations, where the plot of the reconstruction error versus the number of photons per bin (referred to as the imaging efficiency) shows that the Staircase imager needs more photons per bin (or less noise) to reconstruct an image as well as the Michelson imager does.

\section{ACK N O W L E D G M N T S}

This work is part of the SRON activities in developing an imaging mode for the DARWIN nulling array and is a result of the Ph.D. research performed by the first author.

\section{References}

[1] A. Quirrenbach, "Optical interferometry" Ann. Rev. Astron. Astroph., 39, 353-401 (2001).

[2] S.K. Saha, "Modern optical astronomy: technology and impact of interferometry" Rev. Mod. Phys. 74, 551-600 (2002).

[3] J.E. Baldwin and C.A. Haniff, "The application of interferometry to optical astronomical imaging" Phil. Trans. R. Soc. London A 360, 969-986 (2002).

[4] DARWIN The Infrared Space Interferometer: Redbook, ESA$\mathrm{SCl}(2000)$ 12, (2000).

[5] L.A. d'Arcio et al., "The imaging mode of the Infrared Space Interferometer IRSI-Darwin" Proc. of SPIE 4838, 184-195 (2002).

[6] C. van der Avoort, J.W. den Herder, S.F. Pereira and J.J.M. Braat, "Optimum synthetic-aperture imaging of astronomical objects" J. Opt. Soc. Am. A, doc. ID 69867 (posted 10 April 2006, in press).

[7] M.A. Born and E. Wolf, Principles of Optics, Sixth edition, pp. 508510, (Pergamon Press, New York, 1980).

[8] C. van der Avoort, Optical aperture synthesis, Ph.D. thesis, Delft University of Technology, (2006).

[9] J.R.P. Angel, J.M. Hill, P.A. Strittmatter, P. Salinari and G. Weigelt, "Interferometry with the large binocular telescope" Astronomical Interferometry, Proc. of the SPIE 3350, 881-889 (1998).

[10] A. Labeyrie, "Resolved imaging of extra-solar planets with future 10-100km optical interferometric arrays" Astronomy and Astrophysics Supplement 118, 517-524 (1996).

[11] D.T. Leisawitz, B.J. Frey, D.B. Leviton, A.J. Martino, W.L. Maynard, L.G. Mundy, S.A. Rinehart, S.H. Teng and X. Zhang, "Wide-field imaging interferometry testbed I: purpose, testbed design, data, and synthesis algorithms" Interferometry in Space, Proc. of SPIE 4852, Michael Shao ed., 255-267, (2003).

[12] I. Montilla, S.F. Pereira and J.J.M. Braat, "Michelson wide-field stellar interferometry: principles and experimental verification" Appl. 0pt. 44 (3), 328-336 (2005).

[13] J.W. Goodman, Statistical Optics, (John Wiley at Sons, 1985).

[14] W.H. Press, B.P. Flannery, S.A. Teukolsky and W.T. Vetterling, Numerical Recipes in Pascal, (Cambridge University Press, 1989).

[15] J.L. Hora et al., "Calibration and performance of the Infrared Array Camera (IRAC)" SPIE 4131, 13-25 (2000).

[16] G.G. Fazio et al., "The Infrared Array Camera (IRAC) For The Spitzer Space Telescope" The Astrophysical Journal Supplement Series, 154 (1), 10-17 (2004).

[17] J.M. Beckers, "Interferometric imaging with the Very Large Telescope," J. of Optics (Paris) 22 (2), 73-83 (1991).

[18] L.A. d'Arcio, selected aspects of wide-field stellar interferometry, Ph.D. thesis, Delft University of Technology, (1999). 